\title{
Impacts of mutual phase interactions on piezoelectric and dielectric properties
}

\author{
Kun-Ta, Lin, Chia-Hung Pan, Wei-Ting, Li, Jrjeng, Ruan* \\ Department of Materials Science and Engineering, National Cheng Kung University, Taiwan \\ s153126@gmail.com
}

The secondary molecular interactions are well known able to influence the organization behaviours and electrooptical responses of dispersed molecules. [1, 2] For dispersed phase domains of organic and inorganic components, including amorphous and crystalline phases, the mutual polarization is much less recognized. In general, the interactions among phases, specially crystallites, have not been envisaged yet as an approach of crystal engineering and capable factors to enhance the electrooptical features of phase domains.

Ferroelectric polymers are able to evolve unique polar crystalline phase below curie transition temperature via the lattice packing of alltrans conformers, which allocates most of substituted fluorine atoms on one side of molecular segments and hydrogen atoms on the other side. We found that the dielectric and piezoelectric responses of polymer ferroelectric lamellar crystals are significantly enhanced by the nearby $\mathrm{ZnO}$ nanorods crystals, and vice versa. The involved effects of mutual polarization cause both kinds of constituent crystals to adopt opposite polarization orientation. Besides, the extent of mutual interaction will decrease with the distance between nanorods region and PVDF-TrFE lamellae region increase, as shown on Figure 1. This is the first observation of mutual interaction among crystalline phases.
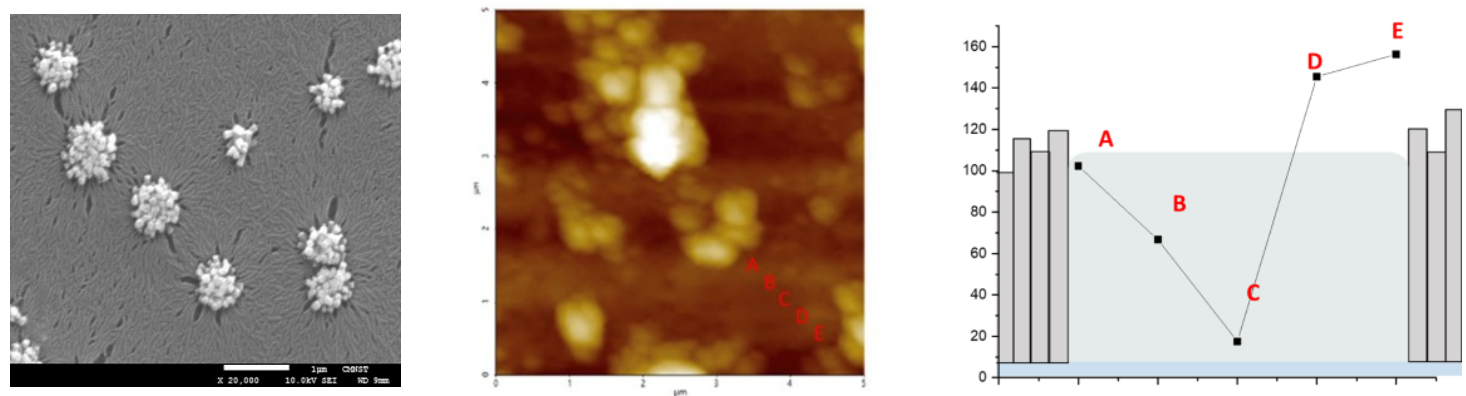

Figure 1. The measurement of $d_{33}$ of PVDF-TrFE ferroelectric crystalline lamellae. As the distance between $\mathrm{ZnO}$ nanorod and lamellar crystals increase, the effect of mutual polarization will be decreased. The presence of $\mathrm{ZnO}$ nanorods is observed as the bright areas due to the height, and the darker regions are caused by the presence of PVDF-TrFE lamellar crystals.

In our recent research success, we found that the stacking of ferroelectric lamellae has impact on piezoelectric and surface potential. With the growth of intact lamellae, the stacking of crystal will be better than the broken ones which is considered corresponding to the alignment of dipole moments. Therefore, the better stacking of lamellae will possess lower surface potential, however the piezoelectric response is lower, as shown on Figure 2. Besides, the mutual phases interactions have been identified between quantum dots and polymer ferroelectric crystals. With the average deposition of graphene and $\mathrm{MoS}_{2}$ quantum dots, the piezoelectricity

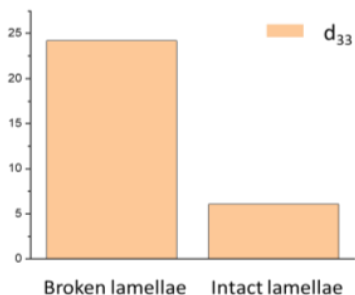

Figure 2. The measurement of $d_{33}$ and surface potential upon the different stacking behaviors of lamellae.

of dispersed ferroelectric polymer crystals has been dramatically enhanced, and graphene quantum dots are able to yield better contribution. This mutual phase interactions among ferroelectric polymer crystals and 2D material quantum dots have been explored as a helpful factor to improve the recombination issue of photocatalysts and therefore enhance the efficiency of water splitting. The involved phase evolution and growth mechanisms have been under investigation, which is expected to serve as a new direction to prepare hybrid crystalline materials able to overcome the current materials application bottlenecks.

[1] Ghosh, T., Panicker, J. S., \& Nair, V. C. (2017). Self-assembled organic materials for photovoltaic application. Polymers, 9(3), 112.

[2] Liu, Y., Song, J., \& Bo, Z. (2021). Designing high performance conjugated materials for photovoltaic cells with the aid of intramolecular noncovalent interactions. Chemical Communications, 57(3), 302-314.

Keywords: mutual phase interactions; quantum dots; Ferroelectric polymers

Financial support by Ministry of Science and Technology (MOST) is gratefully acknowledged (109-2224-E-006-007-).

Acta Cryst. (2021), A77, C997 